\title{
Immature to total neutrophil ratio as an early indicator of early neonatal sepsis
}

\author{
Erum Saboohi' ${ }^{1}$, Farhan Saeed ${ }^{2}$, \\ Rashid Naseem Khan ${ }^{3}$, Muhammad Athar Khan ${ }^{4}$
}

\begin{abstract}
Background \& Objectives: Neonatal septicemia is responsible for 1.5 to 2.0 million deaths/year in the under developed countries of the world. Pakistan is number three among these countries and accounts for $7 \%$ of global neonatal deaths. The objective of the study was to determine the role of simple hematological parameter, immature to total neutrophil ratio (I/T ratio) in diagnosing early onset neonatal bacterial infection.

Methods: A descriptive cross-sectional study was conducted in Neonatal Intensive Care Unit of Liaquat College of Medicine \& Dentistry (LCMD) Hospital from January 2016 to January 2017. A total 85 neonates were admitted with clinical suspicion of presumed early onset sepsis or who had potential risk factors for sepsis like prematurity, prolonged rupture of membranes was carried out. After taking informed consent from parents of admitted neonates, data was collected in a structured questionnaire. Laboratory workup included White blood cell count, CRP, absolute neutrophil count, immature neutrophil count while blood $\mathrm{C} / \mathrm{S}$ was kept as gold standard. Empirical antibiotics started after sample collection for workup. Manual differential count and immature neutrophil count of the peripheral blood smear was performed by a senior technician masked to clinical information. I/T ratio was calculated from WBC, neutrophils and immature neutrophil count by a simple formula.

Results: Out of 85 neonates, 13 had positive blood cultures (15.29\%). The mean white blood count was $18761.18 \pm 8570.75$ and mean I/T ratio was $0.1622 \pm 0.0419$. About $50 \%$ of proven sepsis cases had WBC higher than 26000 as compared to $50 \%$ of cases for negative diagnoses that had WBC $<15500$. The mean I/T in positive CRP $0.204 \pm 0.04$ was non-significantly higher as compared to negative CRP $0.151 \pm 0.034$ $(p=0.084)$. Point biserial correlation revealed that $\mathrm{I} / T$ ratio was significant strong correlation $\left(r_{p b}=0.721\right.$, $\mathrm{p}<0.001)$ and overall I/T ratio was a good indicator of a positive and negative blood culture result. The sensitivity, specificity, positive predictive value (PPV) and negative predictive value (NPV) of I/T ratio were $76.47 \%, 83.82 \%, 54.16 \%$ and $93.44 \%$ respectively. Similarly majority of neonates having high I/T ratio also depicts positive C-reactive protein (CRP) (NPV 91.23\%). Therefore, both I/T and CRP showed a high negative predictive value $(\mathrm{I} / \mathrm{T}=93.44 \%$ and $\mathrm{CRP}=91.23 \%)$ in this study.

Conclusion: I/T ratio is a useful tool for early onset sepsis (EOS) with reasonable specificity but cannot be relied upon as sole indicator. Combination of normal immature to total neutrophil Ratio with negative CRP values in neonates with presumed sepsis is an indicator of non-infected neonate which comprised $78.8 \%$ of our study population.
\end{abstract}

KEYWORDS: Neonatal Sepsis, Early onset sepsis (EOS), CRP, I/T ratio, Blood culture.

doi: https://doi.org/10.12669/pjms.35.1.99

How to cite this:

Saboohi E, Saeed $F$, Khan RN, Khan MA. Immature to total neutrophil ratio as an early indicator of early neonatal sepsis. Pak J Med Sci. 2019;35(1):241-246. doi: https://doi.org/10.12669/pjms.35.1.99

This is an Open Access article distributed under the terms of the Creative Commons Attribution License (http://creativecommons.org/licenses/by/3.0), which permits unrestricted use, distribution, and reproduction in any medium, provided the original work is properly cited.

Correspondence:

Dr. Erum Saboohi, MBBS, MD.

Senior Registrar, Department of Pediatrics,

Liaquat College of Medicine \& Dentistry, Karachi, Pakistan.

E-mail: erumsabohi@yahoo.com

\section{INTRODUCTION}

Neonatal sepsis is described as a suspected or demonstrated infection in newborn, a systemic 
inflammatory response syndrome with variable sign and symptoms caused by pathogens with or without accompanying bacteremia. ${ }^{1}$ The incidence of Neonatal sepsis is approximately 8 per 1000 live births and as high as 13 to 27 per 1000 for newborns weighing < 1500 gms. $^{2}$ Neonatal septicemia is responsible for 1.5 to 2.0 million deaths/year or between 4000 to 5000 deaths/day in the underneath advanced countries of the world. ${ }^{3}$ Two thirds of the world's neonatal deaths occur in just 10 countries, frequently in Asia. Pakistan accounts for $7 \%$ of global neonatal deaths. The predominant causes are infections (36\%), preterm births (28\%) and birth asphyxia (23\%) accounting for about $87 \%$ of neonatal deaths worldwide. ${ }^{4}$

Risk factors for neonatal sepsis are low birth weight infants, birth asphyxia, respiratory compromise at birth, maternal risk factors and congenital anomalies. ${ }^{5}$ Diagnostic tools for identification of EOS includes prenatal screening of high risk mothers to clinical and laboratory identification of newborns with presumed sepsis. Various diagnostic tools have been extensively studied over many years for EOS like WBC, BANDS, ANC, immature to total neutrophil ratio, CRP, interleukin six, procalcitonin.

Blood culture remains a gold standard for diagnosing neonatal sepsis but results are typically obtained after three to five days and its accuracy varies between eight and $73 \%$ in various studies. ${ }^{6}$ However, there are some screening tests (WBC, Platelets, Micro Erythrocyte sedimentation rate (ESR), Absolute Neutrophilic Count (ANC), C-Reactive Protein (CRP), (I/T) ratio, nitroblue teterazolium (NBT), serial Interleukin-6 (IL-6 ) and pro-calcitonin) that could predict sepsis within 6 to 8 hours. ${ }^{7}$ The ANC $(<1000 / \mathrm{ul})$ and the I/T ratio $(\geq 0.2)$ give the clue about the early-onset sepsis in newborns. ${ }^{8}$ Rapid diagnostic tests like CRP, WBC indices may be used as a screening approach for early diagnosis and treatment of sepsis. ${ }^{9}$

In a prospective study by Kredit $\mathrm{T}$ et al in 185 neonates, showed a high NPV of CRP and I/T ratio in early and late onset infection (90\% to $98 \%) .{ }^{10}$ The sensitivity of I/T ratio has ranged from 60 to $90 \%$. Therefore, when diagnosing neonatal sepsis, the elevated I/T ratio values should be considered in conjunction with other clinical signs. ${ }^{11}$ From previous studies it was concluded that I/T ratio of $>0.80$ fairly approximates Neutrophils Storage Pool in bone marrow thereby saving the neonates from bone marrow examinations. This ratio is quiet helpful in identifying newborns who might be benefitted by G-CSF (granulocyte colony stimulating factors). ${ }^{12}$

Early diagnose of EOS by simple hematological ratio can improve clinical outcome in these neonates. ${ }^{13,14}$ The significance of this study is to test the diagnostic accuracy of I/T ratio for EOS before the results of blood culture and sensitivity are available, and from routine $\mathrm{CBC}$ sample collection, important hematological marker I/T ratio can be determined. Reasonable clinical judgment with $\mathrm{I} / \mathrm{T}$ Ratio provides rational basis for treatment decision in neonatal sepsis. Such strategy significantly reduces unnecessary antimicrobial therapies which lengthen hospital stay and can otherwise permit emergence of resistant pathogen strains.

\section{METHODS}

This cross sectional study was conducted to identify and evaluate a scheme for an early detection of neonatal sepsis. Data of 85 infants admitted with presumed sepsis in the NICU of the Liaquat College of Medicine \& Dentistry (LCMD) Hospital, Karachi, from January 2016 to January 2017 was collected and analyzed.

Presumed sepsis was defined if the blood culture was negative but there was a strong clinical suspicion for infection. Neonates with positive blood culture were defined as having confirmed sepsis. The sample size of the study was calculated by WHO sample size calculator ${ }^{15}$ taking sensitivity $75 \%$, specificity $95 \%$, expected prevalence $50 \%$, desired precision $10.5 \%$ and confidence level $95 \%$, the calculated sample size was $(n=85)$.

Leucocyte counts were done on coulter counter in lab. Differential and immature neutrophil counts were performed manually after making suitable thin film smears by senior Lab technician. CRP levels considered negative when $<5 \mathrm{mg} /$ dl .Blood cultures collected with proper a-septic techniques. Liquid thioglycolate lisp(oxoid) was used for blood culture and $2 \mathrm{ml}$ of neonatal blood was added to $20 \mathrm{ml}$ of medium. Inoculated culture bottles were incubated at $36^{\circ} \mathrm{C}$ for seven days and examined for growth every 24 hours; negative culture reports given after 7 days. Blood culture taken as gold standard .Tests used for screening were:

1. total leukocyte counts either less than 5000 or more than 20000/cumm

2. neutropenia/ neutrophilia (age adjusted count, described by Monroe et al 1979) 
3. immature to total neutrophilic count ratio (I/T ratio $>0.2$ )

4. positive $\mathrm{C}$ - reactive protein

Immature to total neutrophil ratio (I/T ratio) calculated by the following formula:

$\%$ of immature neutrophils

$\%$ of total neutrophils(mature +immature)

Statistical analyses were performed using SPSS software version 22 for Windows. Continuous variables like I/T ratio, age, weight, ANC and WBCs were presented as mean and standard deviation, while categorical variables like gender, and distribution of immature Neutrophils were presented as frequencies and percentages. Independent sample t-test was applied to find out the difference among blood culture and study parameters like I/T ratio, age, weight and WBCs. Point biserial correlation was calculated to evaluate the relationship among different study predictors. Chi-square test / Fisher's exact was applied to determine the association of blood culture with $\mathrm{I} / \mathrm{T}$ ratio and CRP. A two-sided $\mathrm{p}$ value $<0.05$ was considered significant.

The current study was approved by the ethical committee of LCMD hospital Karachi. After taking informed consent from parents of admitted neonates, data was collected in a structured questionnaire.

\section{RESULTS}

A total 85 neonates were registered after screening, out of which 48 (56.5\%) were males and $37(43.5 \%)$ were females. The mean age and weight of the neonates were $1.58 \pm 0.76$ days and $2.38 \pm$ $0.55 \mathrm{~kg}$ (maximum $4 \mathrm{~kg}$ and minimum $1.1 \mathrm{~kg}$ ) respectively.

The mean white blood count was $18761.18 \pm$ 8570.75 and mean $\mathrm{I} / \mathrm{T}$ ratio was $0.1622 \pm 0.0419$. ANC ranged between 3100-15000/ul. None of the patient showed neutropenia. The average neutrophil count was $64.88 \pm 10.12$ while immature neutrophil was $10.66 \pm 2.74$. In this study frequency analysis was also performed for WBC count for positive and negative sepsis cases. There was only one case with WBC count $<5000 /$ cumm with proven sepsis. Culture proven sepsis occurred in 15 neonates accounting for $17.64 \%$ of cases. WBC count was abnormal in 10 of 15 culture proven cases $(66.66 \%$ specificity). Elevated I/T ratio $(>0.2)$ identified in 7 of 15 culture proven cases $(46.66 \%$ sensitivity). 55 out of 70 culture negative cases had normal I/T ratio (78.57\% specificity).

Frequency analysis is performed for WBC count for the positive and negative sepsis cases. There is only one case with $<5000$ cumm with proven sepsis. About $50 \%$ of positive cases have WBC higher than 26000 as compared to $50 \%$ of cases for negative diagnoses that have WBC less than 15500/cumm. WBC, together with higher I \T Ratio, could be good indicator for positive diagnosis.

Furthermore, the difference of mean positive blood culture was significantly higher in I/T ratio, age and WBCs as compared negative blood culture. Although, the mean weight with positive blood culture $2.412 \pm 0.618 \mathrm{~kg}$ was slightly more as compared to negative BC $2.382 \pm 0.547 \mathrm{~kg}$ but it was statistically non-significant. The mean I/T in positive CRP $0.204 \pm 0.04$ was non-significantly higher as compared to negative CRP $0.151 \pm 0.034$ $(\mathrm{p}=0.084)$.

Point biserial correlation revealed that $\mathrm{I} / \mathrm{T}$ ratio was significantly correlated with blood CS $\left(\mathrm{r}_{\mathrm{pb}}=\right.$ $0.721, \mathrm{p}<0.001)$ and it was a good indicator of a positive and negative blood culture result (Table-I).

We also observed that the frequency of CRP negative was significantly lower in the patients of positive blood culture $(\mathrm{n}=5,29.4 \%)$ whereas CRP (+ve) in positive blood culture was $(\mathrm{n}=16,23.5 \%)$ $(\mathrm{p}<0.001)$.

Table-I: Correlation of blood culture with I/T ratio, age, weight and total WBC count.

\begin{tabular}{lcccc}
\hline Parameters & $r p b(p$-value $)$ & \multicolumn{2}{c}{ Blood Culture } & P-value \\
\cline { 3 - 4 } & & Negative $(n=68)$ & Positive $(n=17)$ & $<0.001^{*}$ \\
\hline I/T Ratio & $0.721(<0.001)^{*}$ & $0.151 \pm 0.034$ & $0.204 \pm 0.044$ & $0.010^{*}$ \\
Age (days) & $0.280(0.010)^{*}$ & $1.471 \pm 0.722$ & $2.011 \pm 0.791$ & 0.847 \\
Wight (Kg) & $0.021(0.847)$ & $2.382 \pm 0.547$ & $2.412 \pm 0.618$ & $<0.001^{*}$ \\
WBC & $0.488(<0.001)^{*}$ & $16683.831 \pm 7039.878$ & $27070 \pm 9296.220$ & \\
\hline
\end{tabular}

WBC: white blood cell; rbp: Point biserial correlation was calculated for blood culture with all parameters. Continuous variables were expressed as Mean and Standard deviation and Independent sample t-test was applied. *Significant p-value. 
Table-II: Association of Blood Culture with I/T ratio and CRP.

\begin{tabular}{lccc}
\hline Parameters & \multicolumn{2}{c}{ Blood Culture } & $p$-value \\
\hline \multicolumn{3}{c}{ Negative $(n=68)$} & Positive $(n=17)$ \\
\hline I/T Ratio & & & \\
$\geq 0.2$ & $11(16.2 \%)$ & $13(76.5 \%)$ & $<0.001^{*}$ \\
$<0.2$ & $57(83.8 \%)$ & $4(23.5 \%)$ & \\
CRP & & & \\
-VE & $52(76.5 \%)$ & $5(29.4 \%)$ & $<0.001^{*}$ \\
+VE & $16(23.5 \%)$ & $12(70.6 \%)$ & \\
\hline
\end{tabular}

Categorical variables were expressed as Frequency and Percentage. *Significant p-value calculated by Fisher Exact / Chi-square test.

A difference in proportion of $\mathrm{I} / \mathrm{T}$ ratio $\geq 0.2$ was statistically significant higher in positive blood culture $(\mathrm{n}=13,76.5 \% \mathrm{v} / \mathrm{s} \mathrm{n}=11,16.2 \%$ and $\mathrm{p}<$ 0.001 ) in (Table-II). The diagnostic accuracy of $\mathrm{I} / \mathrm{T}$ ratio revealed that sensitivity, specificity, PPV and NPV in neonatal sepsis were $76.47 \%, 83.82 \%$, $54.16 \%$ and $93.44 \%$ respectively (Table-III).

\section{DISCUSSION}

Total 85 neonates with presumed early onset neonatal sepsis (EONS) based on history and examination were investigated. 48 out of 85 admitted neonates with clinical suspicion of early onset sepsis were low birth weight i-e $<2.5 \mathrm{~kg}$ accounting for $56.4 \%$ cases Out of 48 LBW neonates, $30(62.5 \%)$ were preterm ( $<37$ weeks gestation), a finding that was consistent with similar previous studies on neonatal sepsis. ${ }^{16,17}$ There was an inverse relationship of birth weight to infection rate due to impaired cellular immunity in low birth weight neonates which make them more susceptible to acquire infections. ${ }^{18,19}$

About $50 \%$ of positive cases have WBC count $>26000 /$ cumm as compared to $50 \%$ of cases with negative diagnosis that have WBC count $<15500 /$ cumm. Studies have shown that screening WBC count alone are poor predictor of neonatal infection ${ }^{20}$ as some non-infectious conditions also cause neutrophilia like birth asphyxia and stress. CRP showed specificity of $76.47 \%$, sensitivity of $70.58 \%$, PPV of $42.85 \%$ and NPV of $91.23 \%$.

$\mathrm{I} / \mathrm{T}$ ratio reference ranges are obtained from
Schmutz chart. ${ }^{21} \mathrm{~A}$ ratio of $\geq 0.2$ is highly sensitive marker of neonatal septicemia. ${ }^{22}$ In the present study I/T ratio of $\geq 0.2$ showed specificity of $83.8 \%$, sensitivity of $76.5 \%$, PPV of $54.6 \%$ and NPV of $93.44 \%$. Hence, $\mathrm{I} / \mathrm{T}$ ratio of $\geq 0.2$ our results are comparable to other studies ${ }^{22}$. Some previous studies have shown different results in this parameter which may be due to variation in blood sampling, severity of sepsis, age of patient and investigative criteria followed, sensitivity: $90 \%$ to $100 \%$, specificity: $30 \%$ to $78 \%$, PPV: $11 \%$ to $51 \%$ and NPV: $99 \%$ to $100 \% .^{23}$

The present study revealed that, from the various sepsis screen parameters, the I/T ratio showed high specificity and high negative predictive value. The result from this study depict the role of $\mathrm{I} / \mathrm{T}$ ratio as an indicator to exclude early onset neonatal sepsis as negative predictive value is on higher side. This is in agreement with the previous studies which displayed NPV in the range of $90 \%$ to $98 \%$ for I/T ratio $\geq 0.2$ in excluding EOS. Similar study with a NPV of $100 \%$ of I/T ratio $>0.2$ conducted by Murphy et al in 2012 and others. ${ }^{24,25}$

Furthermore, the present study revealed that even simple hematological ratio can help in ruling in or ruling out EOS, thereby decreasing hospital stay, alleviating the anxiety of parents and help preventing development of antibiotic resistant pathogenic strains. This is especially important for the various resources limited clinical setups in underdeveloped countries.

The significance of the study is that it represents a thorough statistical analysis of I $\backslash \mathrm{T}$ Ratio as an early indicator of EONS. This could allow practitioners to take preventive measures at an earlier level. Analysis of other parameters that could contribute to early diagnosis can be advantageous. The statistical significance of other parameters, for example, CRP, preterm births and weights need to be further investigated to establish whether these can improve accuracy of EOS diagnosis when considered together with I $\backslash \mathrm{T}$ Ratio.

Limitations of the study: We had only few infants with positive blood culture. Another important issue when conducting research on sepsis in neonates is that there is no perfect gold standard

Table-III: Diagnostic Accuracy of I/T ratio in cases with EONS.

\begin{tabular}{|c|c|c|}
\hline & Proven Sepsis (CS Positive) & Presumed Sepsis (CS Negative) \\
\hline $\mathrm{I} / \mathrm{T}$ ratio $>0.2$ & $\mathrm{TP}($ true positive $)=13$ & $\mathrm{FP}($ false positive $)=11$ \\
\hline $\mathrm{I} / \mathrm{T}$ ratio $<0.2$ & $\mathrm{FN}($ false negative $)=4$ & $\mathrm{TN}($ true negative $)=57$ \\
\hline
\end{tabular}


with which to compare the results with. When calculating sensitivity and specificity the new result is compared with blood culture, a method with widely known limitations in both sensitivity and specificity. Another limitation is that the study included material from a single tertiary care centre; this increases the risk that the results only reflect the local situation and this must be taken into consideration before the results are generalized to other locations.

\section{CONCLUSION}

Though there are several markers to diagnose neonatal sepsis, the search for the ideal marker is still on. In the present study I $\backslash \mathrm{T}$ Ratio showed high specificity and high negative predictive value for neonatal sepsis. The predictive value of elevated I $\backslash$ $\mathrm{T}$ Ratio and simplicity of the test justifies its routine use in early diagnosis of early onset neonatal sepsis. The markers from the present study and the interpretation from chosen cutoff values ought to be tested in a larger prospective clinical study. Future research in the area of diagnosing neonatal sepsis is highly necessary for the benefit of health care professionals dealing with infected neonates and their attendants.

I/T ratio estimation does have a role in diagnosing early neonatal septicemia but it is not sensitive enough to be relied upon as the sole indicator. When this readily calculated ratio is used together with clinical signs of EONS, a negative test result may help in ruling out EONS. Based on the results of this study, it may be concluded that the early discontinuation of antibiotics (within 24 to 48 hours) in neonates with suspicion of EONS can be planned on $I \backslash T$ Ratio results in conjunction with improving clinical signs. Its usefulness may be enhanced when it is considered in conjunction with other sepsis screen markers like CRP and WBC in ruling out sepsis. More data should be available to draw the final decision. However, we suggest designing a scoring system for the screening of EONS by using combination of tests.

Acknowledgement: The author are grateful to all members of both the NICU and LCMD hospital, hematology laboratory for their meticulous work and great help.

Funding: This research did not receive any specific grant from funding agencies in the public, commercial, or not-for-profit sectors.

Conflict of interest: None.

\section{REFERENCES}

1. Kliegman R, Stanton B, Geme J St, Schor N, (eds). Elsevier, Philadelphia, Nelson's textbook of pediatrics (20th edn.): Infections Neonatal Infant. 2016:909-923.

2. Goldstein B, Giroir B, Randolph A. International pediatric sepsis consensus conference: Definitions for sepsis and organ dysfunction in pediatrics. Pediatr Crit Care. 2005;6(1):28. Copyright 2005, Lippincott Williams \& Wilkins. doi: 10.1097/01.PCC.0000149131.72248.E6.

3. Waseem RI, Khan M, Izhar TS, Qureshi AW. Neonatal sepsis. Professional Med J. 2005;12(4):451-456. Bulletin of the World Health Organization. 2009;87:130-138. doi: 10.2471/ BLT.08.050963.

4. Tricia Lacy Gomella; M. Douglas Cunningham; Fabien G. Eyal. Neonatology $7^{\text {th }}$ edition: Sepsis. 2013:865-874. ISBN 10: 0071768017 ISBN 13: 9780071768016. Publisher: McGraw-Hill Education/ Medical.

5. Simonsen KA, Anderson-Berry AL, Delair SF, Davies HD. Early-Onset Neonatal Sepsis. Clin Microbiol Rev. 2014;27(1):21-47. doi: 10.1128/CMR.00031-13.

6. Hornik CP, Benjamin DK, Becker $\mathrm{KC}$, Jennifer $\mathrm{Li}$, Clark RH, Wolkowiez MC, et al. Use of the Complete Blood Cell Count in Early-Onset Neonatal Sepsis. Pediatr Infect Dis J. 2012;31(8):799-802. doi: 10.1097/ INF.0b013e318256905c.

7. Newman TB, Draper D, Puopolo KM, SooraWi, Escobar GJ. Combining Immature and Total Neutrophil Counts to Predict Early Onset Sepsis in Term and Late Preterm Newborns: Use of the $\mathrm{I} / \mathrm{T}^{2}$. Pediatr Infect Dis J. 2014;33(8):798-802. doi:10.1097/INF.0000000000000297.

8. Monica L, Riti JS, Amit BK. Role of Sepsis Screen Parameters in Early Diagnosis of Neonatal Septicemia. Int J Curr Microbiol App Sci. 2018;7(1):2410-2419. doi: 10.20546/ ijcmas.2018.701.290.

9. Arnon S, Litmanovitz. Diagnostic tests in neonatal sepsis. Curr Opin Infect Dis J. 2008;21(3)223-227. doi: 10.1097/ QCO.0b013e3282fa15dd.

10. Krediet T, Gerards L, Fleer A, van Stekelenburg G. The predictive value of $\mathrm{C}$-reactive protein and I/T-ratio in neonatal infection. J Perinat Med. 1992;20(6):479-485.

11. Bhandari V. Effective Biomarkers for Diagnosis of Neonatal Sepsis. J Pediatr Infect Dis Soc. 2014;3(3):234-245. doi: 10.1093/jpids/piu063.

12. Wheeler JG, Chauvenet AR, Johnson CA, Dillard $\mathrm{R}$, Block SM, Boyle R, et al Neutrophil storage pool depletion in septic, neutropenic neonates. Pediatr Infect Dis. 1984;3(5):407-409.

13. Oeser C, Lutsar I, Metsvaht T, Turner MA, Heath PT, Sharland M. Clinical trials in neonatal sepsis. J Antimicrobial Chemotherapy. 2013;68(2):2733-2745. doi: $10.1093 / \mathrm{jac} / \mathrm{dkt} 297$.

14. Panwar C, Kaushik SL, Kaushik R, Sood A. Correlation of neonatal and maternal clinico-hematological parameters as predictors of early onset neonatal sepsis, Int J Contemp Pediatr. 2017;4(1):36-42. doi: 10.18203/2349-3291. ijcp20164516.

15. Naing NN. Determination of Sample Size. Malays J Med Sci. 2003;10(2):84-86.

16. Ng PC, Lam HS. Diagnostic markers for neonatal sepsis. Curr Opin Pediatr. 2006;18(2):125-131.

17. Stoll BJ, Hansen NI, Sanchez PJ, Faix RG, Poindexter BB, Van Meurs KP, et al. Early Onset Neonatal Sepsis. The Burden of Group B Streptococcal and E. coli Disease Continues. Pediatrics. 2011;127(5):817-826. doi: 10.1542/ peds.2010-2217. 
18. Bhutta ZA, Yusuf K. Early-onset neonatal sepsis in Pakistan: a case control study of risk factors in a birth cohort. Amer J Perinatol. 1997;14(9):577-581.

19. Hornik CP, Fort P, Clark RH, Watt K, Benjamin DK Jr, Smith $\mathrm{PB}$, et al. Early and late onset sepsis in very-low-birth-weight infants from a large group of neonatal intensive care units. Early Hum Dev. 2012;88(Suppl 2):S69-74. doi: 10.1016/ S0378-3782(12)70019-1.

20. Hornik CP, Benjamin DK, Becker KC, Benjamin DK, Li J, Clark RH, et al. Use of the Complete Blood Cell Count in Early-Onset Neonatal Sepsis. Pediatr Infect Dis J. 2012;31(8):799-802. doi: 10.1097/INF.0b013e318256905c.

21. Schmutz N, Henry E, Jopling J, Christensen RD. Expected ranges for blood neutrophil concentration of neonates: The Manroe and Mouzinho charts revisited. J Perinatol. 2008;28(4):275-281.

22. Desai P, Shah AN, Pandya T, Desai P, Pandya T. C-Reactive protein, Immature to total Neutrophil Ratio and Micro ESR in early diagnosis of Neonatal Sepsis. Int J Biomed Adv Res. 2014;5(8):364-366. doi: 10.7439/ijbar.v5i8.830

23. Gerdes JS. Diagnosis and management of bacterial infections in the neonate. Pediatr Clin North Am. 2004;51(4):939-959. doi: $10.1016 /$ j.pcl.2004.03.009
24. Murphy K, Weiner. Use of leukocyte counts in evaluation of early-onset neonatal sepsis. J Pediatr Infect Dis. 2012;31(1):16-19. doi: 10.1097/INF.0b013e31822ffc17.

25. Camacho-Gonzalez A, Spearman PW, Stoll BJ. Neonatal infectious diseases: evaluation of neonatal sepsis. Pediatr Clin North Am. 2013;60:367-389. doi: 10.1016/j. pcl.2012.12.003.

\section{Author's Contribution:}

ES: Conceptualization and preparation of manuscript.

FS: Data collection and manuscript writing. RNK: Design, Review and final approval of manuscript.

MAK: Write up, statistical analysis, review.
Authors:

1. Dr. Erum Saboohi, MBBS, MD. Senior Registrar, Department of Pediatrics

2. Dr. Farhan Saeed, MBBS, DCH, FCPS. Assistant Professor, Department of Pediatrics

3. Dr. Rashid Naseem Khan, MBBS, MD, MCPS. Principal (Medical),

4. Dr. Muhammad Athar Khan, MBBS, MCPS, MBA, DPH, DCPS-HPE, DCPS-HCSM, PGD-Statistics, CCRP. Associate Professor, Department of Community Medicine,

1-4: Liaquat College of Medicine \& Dentistry, Karachi, Pakistan.

Correspondence:

Dr. Erum Saboohi, MBBS, MD. Senior Registrar,

Department of Pediatrics, Liaquat College of Medicine \& Dentistry, Karachi, Pakistan.

E-mail: erumsabohi@yahoo.com

* Received for Publication: June 27, 2018

* $1^{\text {st }}$ Revision Received:

* $2^{\text {nd }}$ Revision Received:

* $3^{\text {rd }}$ Revision Received:

* Final Revision Accepted:
July 20, 2018

August 4, 2018

November 5, 2018

November 8, 2018 